\title{
Estratégia\&Negócios
}

ISSN 1984-3372

http://www.portaldeperiodicos.unisul.br/index.php/EeN/

\section{A CAPACIDADE DE PRODUÇÃO COMO RECURSO GERADOR DE GANHOS}

\section{PRODUCTION CAPACITY AS A REMEDY FOR PROFIT GENERATOR}

\section{Norberto Tamborlin}

Mestrando em Administração pela Universidade Regional de Blumenau - FURB

E-mail: norberto.mestrado@gmail.com

\section{Cristian Tadeu Von Der Hayde}

Mestrando em Administração pela Universidade Regional de Blumenau - FURB.

E-mail: cristian.heyde@gmail.com

\section{Camila da Silva Schmitt}

Mestrando em Administração pela Universidade Regional de Blumenau - FURB.

E-mail: camila.s.schmitt@gmail.com

\section{Gabriele Heiden}

IBES/SOCIESC - Instituto Blumenauense de Ensino Superior.

E-mail: norbin@unibes.edu.br

\section{Oscar Dalfovo}

Professor Titular da Fundação Universidade Regional de Blumenau- FURB.

E-mail: odalfovo@gmail.com

Recebido em 05/08/2012. Aprovado em 07/01/2013. Disponibilizado em 04/03/2013. Avaliado pelo Sistema double blind review

R. eletr. estrat. neg., Florianópolis, v.5, n.3, p.28-56, set./dez. 2012 http://portaldeperiodicos.unisul.br/index.php/EeN/index
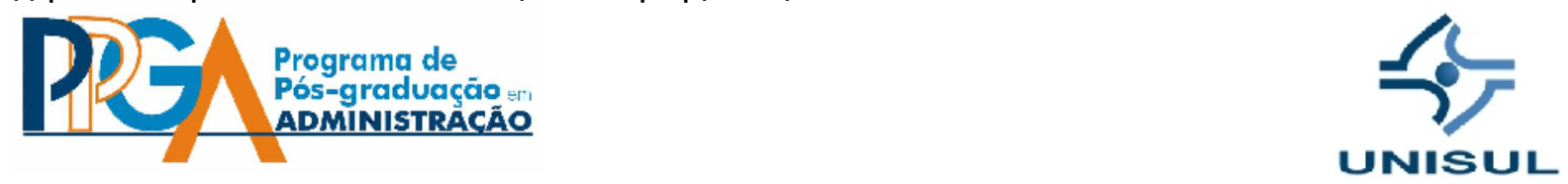

CCopyright 2008 UNISUL-PPGA/Estratégia e Negócios. Todos os direitos reservados. Permitida citação parcial, desde que identificada a fonte. Proibida a reprodução total. Em caso de dúvidas, consulte o editor:

ademar.unisul@gmail.com; (48) 3229-1932

R. eletr. estrat. neg., Florianópolis, v.5, n.3, p. 28-56, set./dez. 2012 


\section{RESUMO}

O crescimento gradativo do mercado de flexografia gera diversas expectativas nas organizações deste segmento. Para garantir o atendimento da demanda e garantir a sua fatia de mercado, é importante conhecer a real capacidade produtiva da sua unidade de produção. A pesquisa teve como objetivo identificar a capacidade de produção do setor de gravação de uma clicheria da cidade de Blumenau/SC. Foi definida como pesquisa qualitativa, de objetivo descritivo e quanto aos procedimentos de investigação, a pesquisa se caracterizou como pesquisa-ação. Os dados foram coletados a partir do acompanhamento diário dos processos realizados no setor de gravação, observação do desenvolvimento das atividades, registro das informações em relatórios e entrevistas informais com os funcionários. Dessa forma, os dados obtidos foram triangulados e apresentados em figuras e tabelas. Como resultados desta investigação, foi identificado o gargalo de produção, bem como a existência de horas paradas programadas por falta de serviço, sendo feitas sugestões para otimizar a capacidade produtiva, assim como estratégias para aumentar os ganhos.

Palavras-chave: Flexografia. Tempo Padrão. Capacidade Produtiva. Restrição do sistema.

\section{INTRODUÇÃO}

O contexto atual da flexografia no Brasil demonstra um mercado que está crescendo gradativamente, principalmente no segmento de embalagens flexíveis.

A clicheria de Blumenau estudada oferece ao mercado os serviços de préimpressão, que englobam o atendimento técnico ao cliente, o desenvolvimento de artefinal, layout e reestruturação de arquivos, o tratamento de imagem, a editoração, o gerenciamento de cores, gerenciamento de arquivos, a prova impressa digital e no substrato, assistência técnica, o sistema de gravação digital e o controle de qualidade.

O objeto de estudo desta pesquisa foi o setor de gravação, que é responsável pela gravação dos arquivos em chapas flexíveis de fotopolímero, denominadas de matrizes de impressão com a função de transferir a tinta para o substrato. Essa pode ser considerada 
como a última etapa do processo de pré-impressão. O setor de gravação depende da entrada de novos pedidos e da capacidade produtiva das etapas anteriores à gravação, porém, esse setor será isolado dos demais com o intuito de calcular a capacidade de produção apenas desse departamento. Atualmente, esse setor tem sua equipe composta por dois gravadores e três auxiliares de gravação, que trabalham dois no turno geral e três no turno noturno. O setor de gravação possui duas máquinas gravadoras, uma expositora, uma lavadora, o forno e os processos de acabamentos.

Foram medidos os tempos dos processos de gravação, que incluem leitura da ordem de serviço, imposição dos arquivos nas chapas, determinar a camada de gravação, acionar marca de refile, selecionar a matéria-prima, exposição de verso, fixação da chapa na gravadora, iniciar a gravação, exposição de frente, lavação do clichê, secagem do clichê no forno, light finish (exposição em luz UVC e UVA), registrar dados da gravação no sistema (data de gravação, utilização de chapas inteiras ou retalhos, geração de retalhos e lixo) e acabamento que incluem refile dos clichês, conferência das especificações da ordem de serviço com o clichê físico, medição da espessura e emissão do laudo de qualidade e, se necessário, solicitar a regravação de arquivo com as devidas correções.

Existe uma discrepância entre a saída de arquivos editorados e a entrada dos pedidos na gravação. Com base nisso, a ideia central foi isolar o setor de gravação e calcular a capacidade produtiva apenas dessa área da empresa, como se todos os serviços recebidos pelos clientes já viessem prontos para gravar ou então que o ritmo produtivo estivesse sempre em constância, ou seja, sem atrasos nas entradas de arquivos para a gravação, a fim de que se possa constatar a capacidade produtiva total e qual a capacidade de produção que está sendo utilizada.

O crescimento do mercado flexográfico implica em estar preparado para suprir o consequente aumento da demanda. Então, para garantir sua fatia de mercado é fundamental para a clicheria Blumenau conhecer a capacidade de produção do setor de gravação, o que além de fornecer subsídios para a tomada de decisão, permitirá aproveitar melhor a mão de obra e otimizar o tempo de execução das atividades. 
Este trabalho tem relevância social para a empresa estudada, pois fornece dados acerca da sua capacidade de produção, informação que propiciará subsídios para a tomada de decisão. Quanto à sua relevância científica, pode servir de base para estudos acadêmicos e na elaboração de futuras monografias e pesquisas.

\section{FUNDAMENTAÇÃO TEÓRICA}

Este capítulo tem como objetivo desenvolver o referencial teórico, fundamentado na ideia de diversos autores. Para tanto, foi dividido em sistema de produção, capacidade de produção e tempo padrão, sendo estes os principais fatores abordados para o desenvolvimento deste trabalho.

\subsection{SISTEMA DE PRODUÇÃO}

Um sistema de produção é definido como um conjunto de atividades e operações envolvidas no processo de produção de bens e serviços. O conjunto de atividades e operações está vinculado à utilização de insumos, que consistem em recursos (matériaprima, mão de obra, capital, máquinas e equipamentos, instalações e conhecimentos técnicos de processos) a serem utilizados para as transformações de produtos. Esse processo de conversão resulta na modificação das matérias-primas e a forma de seus recursos (MOREIRA, 2004).

A função produção em uma organização é visualizada como o conjunto de recursos destinados à produção de bens e serviços. Uma produção eficaz utiliza seus recursos de forma que os bens e serviços produzidos atendam e satisfaçam as necessidades dos seus consumidores. Nessa mesma linha de raciocínio, a produção eficaz ao mesmo tempo em que proporciona vantagens competitivas para as organizações, também fornece meios para o atendimento dos objetivos estratégicos de longo prazo (SLACK et al., 2008). As organizações existem com o objetivo de gerar recursos, assim a finalidade está em produzir ou transformar recursos em outros bens ou serviços com maior valor agregado. 
Nesse processo de buscar um melhor aproveitamento dos recursos é importante destacar o papel da melhoria contínua nos processos produtivos. Para Caffyn (1999), a melhoria contínua é o envolvimento total na realização de pequenas transformações de melhoria que são realizadas de uma forma contínua e estão diretamente relacionadas aos objetivos da organização. Leede e Looise (1999) afirmam que a melhoria contínua é vital em um ambiente competitivo. Imai (1986), Berger (1997) apresentam os princípios fundamentais da melhoria contínua que consideram importantes: orientação ao processo; pequenas melhorias no padrão de trabalho; e orientação ao indivíduo. Mas, a afirmação de Savolainen (1998) e Bessant e Francis (1999) de que o processo de melhoria contínua é um processo de aprendizagem que não ocorre da noite para o dia, deixa claro que essas melhorias são pontuais e ocorrem devido a uma aprendizagem prática.

\subsection{PRODUÇÃO NO SERVIÇO}

A administração de serviços está relacionada ao que está sendo oferecido e como é realizada a prestação para o cliente. Engloba o entendimento das necessidades do cliente, o gerenciamento, bem como assegurar que os objetivos sejam atendidos e aprimorar continuadamente os serviços (JOHNSTON, 2002).

A literatura de gestão é quase unânime em sugerir aos fabricantes de produtos para integrar os serviços em suas ofertas do produto principal, por exemplo, Bowen et al. (1991), Gadiesh e Gilbert (1998), Quinn et al. (1990), Wise e Baumgartner (1999). A justificativa para essa integração é normalmente colocada de três modos. Primeiro, há argumentos econômicos. Receitas substanciais podem ser geradas a partir de uma base instalada de produtos com um ciclo de vida longo (KNECHT et al., 1993; POTTS, 1988); os serviços, em geral, possuem margens superiores em relação aos produtos (ANDERSON et al, 1997). Além disso, proporcionam uma fonte de receitas mais estável à medida que são resistentes aos ciclos da economia, ao investimento na unidade de fabricação e compra de equipamentos (QUINN, 1992). 
Em segundo lugar, clientes estão demandando mais serviços. A pressão para reduzir o tamanho de criar mais flexibilidade, definir mais claramente as competências essenciais e crescentes da complexidade tecnológica que conduz a uma elevada especialização são algumas das forças motrizes por trás do aumento da terceirização de serviços (LOJO, 1997).

Finalmente, há o argumento competitivo. Serviços, por serem menos visíveis e mais dependentes de pessoas, é muito mais difícil de copiar, tornando-se assim uma sustentável fonte de vantagem competitiva (HESKETT et al., 1997).

Martins e Laugeni (2006) afirmam que o setor de serviços corresponde a uma maior parcela do produto interno bruto e atualmente emprega um maior número de pessoas. Sobre isso, Corrêa e Corrêa (2008) argumentam que os serviços adquiriram grande importância para a economia. Atualmente eles representam uma arma competitiva no mercado globalizado, visto que a diferenciação não está apenas nas características do produto e sim está incorporado ao atendimento recebido pela organização.

Serviço é definido como uma combinação dos resultados e experiências proporcionadas ao cliente e recebidos por esse mesmo cliente (JOHNSTON, 2002). Com base nessas considerações, nota-se que o cliente acaba julgando a qualidade do serviço tanto pela experiência quanto pelo resultado.

Para Barnes et al. (1986), o conceito de serviço está relacionado ao modo como a organização gostaria de ter seus serviços percebidos por seus clientes, funcionários, acionistas e financiadores. Dessa forma, Corrêa e Corrêa (2008) enfatizam que a produção de serviços apresenta diferenças básicas se comparadas com a produção de produtos, primeiramente, os serviços não podem ser estocados nem transportados e há uma evidente necessidade de interação com o consumidor.

Produção está relacionada à transformação de insumos em produtos e serviços enquanto a produtividade está vinculada a melhor ou a pior utilização desses recursos. 


\subsection{CAPACIDADE}

A capacidade é o ritmo máximo de produção de um processo (RITZMANN; KRAJEWSKI, 2004). Para Moreira (2004), é a quantidade máxima de produtos e/ou serviços que podem ser produzidos numa unidade produtiva. Conforme Slack et al. (2008), capacidade envolve o fornecimento de produtos e serviços e demanda do mercado. Então, identificar a capacidade produtiva fornece à organização meios para saber se a sua capacidade poderá suprir a demanda do mercado.

Capacidade de produção é o máximo que a organização consegue produzir, enquanto o volume de produção está relacionado à quantidade produzida atualmente (MARTINS; LAUGENI, 2006). Estudos de mercado e previsão de demanda são ferramentas que subsidiam as decisões sobre a capacidade necessária futuramente para uma unidade produtiva. E isso influencia diretamente no planejamento das instalações, planejamento das necessidades de mão de obra e equipamentos.

Segundo Moreira (2004), existe uma relação entre capacidade e custos operacionais. Ele explica que no momento que a capacidade se iguala à demanda, não há excesso de custos. $\mathrm{O}$ excesso de custo ocorre quando a capacidade supera ou é menor que a demanda. Ainda finaliza que o estudo da capacidade é essencialmente estratégico, e que reflete no envolvimento de grandes somas de capital, imobilização de recursos, dificuldade de mudança e impactos nos custos de operação.

Quando a capacidade de produção atinge seu ponto máximo é denominado pico de capacidade. O pico de capacidade pode ser mantido durante algum momento do dia, ou período do mês (RITZMANN; KRAJEWSKI, 2004). Martins e Laugeni (2006) partem da mesma ideia e afirmam que pico de capacidade corresponde à máxima capacidade em condições ideais, ou seja, sem sobrecarregar os recursos internos, dentro de um curto espaço de tempo.

A capacidade da produção é dividida em três partes: capacidade do projeto, capacidade efetiva e capacidade real. A capacidade do projeto ou teórica é a capacidade que o produto tem baseada nas informações do fornecedor ou fabricante (MARTINS; LAUGENI, 
2006). Conforme Slack et al. (2008), a capacidade do projeto corresponde ao que foi projetado para a operação e que nem sempre funciona na prática. Já a capacidade efetiva é a produção máxima que um processo ou uma organização mantém economicamente em condições normais, ou seja, atuando com horários de trabalho realistas e equipamentos necessários instalados (RITZMANN; KRAJEWSKI, 2004).

Martins e Laugeni (2006) complementam que a capacidade efetiva é o que o equipamento apresenta depois de descontar todas as horas técnicas paradas, que envolvem o tempo necessário para manutenção, preparação, processo, limpeza e entre outros. Slack et al. (2008) define que as perdas se dão em casos de mudanças de produtos, manutenção dos equipamentos e entre outros. Após deduzir essas perdas resta a capacidade efetiva da operação. Por fim, o volume de produção real consiste em deduzir também as perdas que não fazem parte do processo produtivo a exemplo de problemas com a qualidade. Segundo Corrêa e Corrêa (2008), a capacidade real é identificada após deduzir as perdas evitáveis. Por exemplo, a quebra por falta de manutenção ou por perdas inevitáveis, como a troca de turno e mudança de produto.

Além do cálculo das capacidades, ainda é necessário calcular a utilização e a eficiência. Slack et al., (2008) define que a proporção entre a capacidade do projeto e a capacidade efetiva resulta na utilização. O resultado é obtido por meio da fórmula:

Figura 1 - Cálculo de utilização

Utilização: Volume de produção real Capacidade do projeto

Fonte: Slack et al., (2008).

O resultado da utilização dá a ideia de quanto da capacidade do projeto está sendo utilizada baseada na capacidade real (CORRÊA; CORRÊA, 2008). E também se calcula a porcentagem de eficiência, por meio da seguinte fórmula:

Figura 2 - Cálculo de eficiência

Eficiência: Volume de produção real

Capacidade efetiva

Fonte: Corréa e Corrëa (2008). 
A partir disso, o cálculo da eficiência propicia identificar qual a real eficiência de projeto, pois deduz todas as perdas evitáveis e inevitáveis em relação à capacidade efetiva (CORRÊA; CORRÊA, 2008).

\subsubsection{Tempo padrão}

Segundo Martins e Laugeni (2006), um dos métodos mais utilizados pelas organizações para mensurar o tempo de trabalho, são as cronometragens, cujo objetivo é identificar a eficiência individual e estabelecer o tempo padrão. O cálculo do tempo padrão fornece dados importantes para a organização ao:

a) estabelecer padrões nos programas de produção que permitem planejar a capacidade da fábrica, de modo que os recursos disponíveis sejam utilizados de maneira eficaz e também permite comparar o desempenho atingido com o padrão existente;

b) fornecer dados para definir os custos padrões, levantar os custos de fabricação, elaboração de orçamentos e estimar custos de novos produtos;

c) prover dados para o equilíbrio da estrutura de produção e planejamento da capacidade.

Já Moreira (2004) afirma que o cálculo do tempo padrão possui duas grandes utilidades:

a) utilidade para estudos futuros que objetivem calcular o custo industrial de um certo produto;

b) utilidade para avaliação, em caso de redução ou não do tempo padrão, para identificar se houve melhorias nos métodos de trabalho.

Slack et al. (2008) sustenta que o estudo do tempo é uma técnica de medida do trabalho para registrar os tempos e o ritmo de trabalho, realizada sob condições 
especificadas, e também para analisar os dados de forma a obter o tempo necessário para a realização de um trabalho com um nível definido de desempenho.

Slack et al. (2008) separam a definição de tempo básico e tolerância:

a) Tempo básico: tempo levado por um trabalhador qualificado, que faz um trabalho especificado com desempenho-padrão;

b) Tolerância: concessões acrescentadas ao tempo básico para permitir descanso, relaxamento e necessidades pessoais.

Moreira (2004) defende que para se estabelecer o tempo padrão é preciso identificar o tempo real e o tempo normal:

a) Tempo real: obtido por meio de cronometragem direta do operador, e varia de operador a operador, bem como para o mesmo operador em situações distintas. São necessárias várias medições a fim de tornar confiável o resultado final, o que resultará no tempo normal;

b) Tempo normal: tempo demandado para completar uma operação com velocidade normal, ou seja, manter a eficiência média durante o trabalho, sem fadiga indevida.

Conforme Slack et al., (2008) para se obter o tempo básico é necessário seguir três etapas:

a) Observar, medir e avaliar: deve-se cronometrar o tempo de realização da atividade; nesta etapa é avaliada a velocidade de trabalho do trabalhador;

b) Ajustar os tempos observados: consiste na relação avaliação observada / avaliação-padrão;

c) Calcular a média dos tempos básicos: baseada na ideia de que o ser humano não consegue repetir uma atividade exatamente no mesmo tempo em todas as ocasiões; é realizado o cálculo da média dos tempos. 
Moreira (2004) relata que os estudos de tempos, requerem algumas providências básicas:

a) a fim de evitar desconfianças e ressentimentos, deve-se comunicar ao operador a necessidade de realização do estudo;

b) conhecer profundamente as operações, e isso envolve o local onde ela é desenvolvida, os equipamentos utilizados e as condições na qual o trabalho é realizado;

c) no caso da atividade ser muito longa, deve-se dividi-la em partes menores.

\section{METODOLOGIA}

Neste capítulo foram apresentados os procedimentos metodológicos utilizados para analisar como a capacidade produção pode auxiliar nos ganhos da Clicheria Blumenau Ltda.

\subsection{MODALIDADE DE PESQUISA}

Quanto à natureza dos dados, esta pesquisa se enquadra como qualitativa. $\mathrm{Na}$ visão de Richardson (1989), a metodologia qualitativa se caracteriza como a tentativa de uma compreensão detalhada dos significados e características apresentados pelos entrevistados, em lugar da produção de medidas quantitativas de características ou comportamentos.

Quanto aos objetivos, a pesquisa se caracteriza como descritiva que, conforme Gil (2006) tem como objetivo principal promover a descrição das características de um determinado grupo, população, fenômeno ou experiência. Ainda conforme o autor, a pesquisa descritiva consiste em analisar dados já conhecidos e tem como principal contribuição a criação de novas visões sobre essa realidade que já se conhece. 
Quanto aos procedimentos de investigação, o estudo se encaixa como pesquisaação. A definição de pesquisa-ação segundo Thiollent (1987) é um tipo de pesquisa social com base empírica que é concebida e realizada em estreita associação com uma ação ou com a resolução de um problema coletivo, nos quais os pesquisadores e os participantes representativos da situação ou do problema estão envolvidos do modo cooperativo ou participativo.

Com base nisso, a pesquisa-ação está atrelada ao desenvolvimento de uma pesquisa, bem como ao planejamento de uma ação, e é caracterizada pelo envolvimento tanto dos pesquisadores quanto dos pesquisados no processo de pesquisa.

\subsection{CAMPO DE OBSERVAÇÃO}

Os estudos relacionados a este trabalho foram realizados na matriz de uma clicheria na cidade de Blumenau. A abrangência deste trabalho se restringe ao setor de gravação. O quadro funcional é composto atualmente por dois gravadores e três auxiliares de gravação. Quanto ao maquinário envolvido no processo, a empresa possui duas máquinas gravadoras, uma expositora, uma lavadora, um forno e os processos de acabamentos.

\subsection{INSTRUMENTO DE COLETA DE DADOS}

Os dados necessários para a elaboração deste estudo foram coletados por meio do acompanhamento diário dos processos, observação do desenvolvimento das atividades, registros das informações obtidas por meio de relatórios e entrevistas informais com os funcionários.

De acordo com Gil (2006), a observação é uma técnica que utiliza os sentidos com a finalidade de adquirir o conhecimento necessário sobre o processo. 0 tipo de observação adequado à pesquisa é a do tipo sistemática, pois está relacionado à elaboração de um documento antecipadamente à coleta de dados, em que serão registrados e interpretados os dados obtidos. As entrevistas são ferramentas que coletam informações 
por meio da formulação de perguntas do entrevistador ao entrevistado, e é vista como uma forma de interação social, em que uma parte deseja coletar dados e a outra parte é a fonte de informações. A entrevista informal se caracteriza pela pouca ou nenhuma estruturação e somente se distingue da simples conversação devido ao objetivo que é a coleta de dados.

\subsection{CRITÉRIOS PARA ANÁLISE DOS DADOS}

Os dados coletados foram triangulados e apresentados na forma descritiva, em tabelas e quadros. Inicialmente foi calculado o tempo padrão do processo de gravação, subdividido em etapas. Definido critérios (tipos de produto) para a elaboração do cálculo do tempo padrão, foram levantadas também as horas paradas programadas e não programadas do processo. A partir desse ponto, foi então calculada a capacidade de produção do setor de gravação. Ao final, foi feito um comparativo entre o desempenho que estava sendo realizado e o desempenho que deveria estar sendo realizado.

\section{RESULTADOS E ANÁLISES}

Neste capítulo serão apresentados os dados obtidos para a solução de cada objetivo específico.

\subsection{CÁLCULO DO TEMPO PADRÃO}

Com a finalidade de calcular o tempo padrão do setor de gravação, todos os funcionários foram comunicados sobre a realização da pesquisa, sobretudo do viés acadêmico que ela propõe, bem como a proposta de otimizar o processo produtivo. 0 processo de gravação foi dividido em dez etapas principais. Cada etapa foi estudada e teve o tempo de processo cronometrado, combinando com as ideias de Martins e Laugeni (2006) que afirmam que um dos métodos mais utilizados pelas organizações para mensurar o tempo de trabalho são as cronometragens, cujo objetivo é identificar a eficiência individual e 
estabelecer o tempo padrão. Sem deixar de refletir o pensamento de Moreira (2004) que relata que para calcular o tempo padrão é importante comunicar ao operador sobre a realização do estudo. Assim, evita-se desconfianças e ressentimentos. Também é necessário conhecer profundamente as operações, o local onde ela é desenvolvida, os equipamentos utilizados e as condições na qual o trabalho é realizado. No caso da atividade ser muito longa, deve-se dividi-la em partes menores.

Na Tabela 1 está descrita a divisão das medições realizadas no setor de gravação para elaboração do cálculo do tempo padrão.

Tabela 1 - Processo de gravação em dez etapas

\begin{tabular}{|c|c|}
\hline Etapas & Descrição \\
\hline $1 \underline{a}$ & Tempo antes da gravação \\
\hline $2 \underline{a}$ & Tempo de gravação \\
\hline $3 \underline{a}$ & Tempo de exposição de frente \\
\hline $4 \underline{a}$ & Tempo de lavação dos clichês \\
\hline $5 \underline{a}$ & Tempo de secagem dos clichês \\
\hline 6a & Tempo de descanso \\
\hline 7a & Tempo de Light Finish \\
\hline $8 \underline{a}$ & Tempo de refile \\
\hline 9a & Tempo de conferência \\
\hline $10 \underline{a}$ & Tempo de fechamento do pacote \\
\hline
\end{tabular}

Fonte: Elaborado pelos autores, 2012.

Foram selecionadas entre os meses de junho até agosto do ano corrente (2011) cem medições das etapas em que o tempo é variável (ou seja, fatores como quantidade de arquivos por chapa podem interferir no tempo de processo), separando as medições por tamanho de chapa, e foi relacionado também os tempos padrão de máquina, que variam de acordo com a marca e espessura da chapa. Os tempos padrão de máquina estão

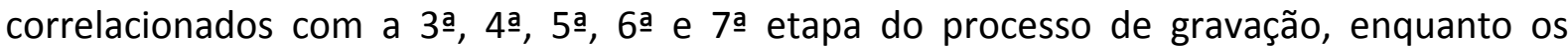

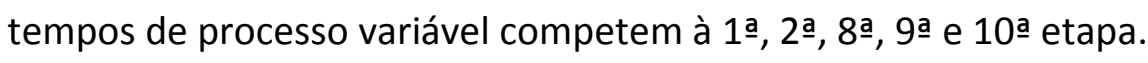

Na Tabela 2 estão dispostos os tempos padrão de máquina para cada uma das etapas em que o tempo varia conforme marca e espessura. 
Tabela 2 - tempo padrão de máquina

\begin{tabular}{lccccc}
\hline \multicolumn{1}{c}{ Espessura/marca } & Etapa 3 & Etapa 4 & Etapa 5 & Etapa 6 & Etapa 7 \\
\hline 0,76- MacDermid & $00: 10: 00$ & $00: 04: 00$ & $01: 15: 00$ & $00: 05: 00$ & $00: 02: 00$ \\
1,14 - Dupont & $00: 10: 00$ & $00: 04: 00$ & $01: 15: 00$ & $00: 05: 00$ & $00: 06: 00$ \\
1,14- MacDermid & $00: 10: 00$ & $00: 04: 00$ & $01: 15: 00$ & $00: 05: 00$ & $00: 06: 00$ \\
1,70- Dupont & $00: 10: 00$ & $00: 07: 30$ & $01: 15: 00$ & $00: 05: 00$ & $00: 07: 00$ \\
1,70- MacDermid & $00: 10: 00$ & $00: 07: 30$ & $01: 15: 00$ & $00: 05: 00$ & $00: 07: 00$ \\
2,84- Dupont & $00: 13: 00$ & $00: 10: 00$ & $01: 15: 00$ & $00: 05: 00$ & $00: 15: 00$ \\
\hline
\end{tabular}

Fonte: Elaborado pelos autores, 2012.

A partir dos tempos médios obtidos na pesquisa, identificou-se a necessidade de estabelecer critérios para a elaboração do cálculo do tempo padrão. Os critérios levaram em consideração o tamanho da chapa (jumbo, média ou retalho), espessura do material $(0,76$, 1,14, 1,70 ou 2,84 mm), a marca (Dupont ou MacDermid), a gravadora (CDI Wide TermoFlex ou CDI Spark 5080) e a quantidade de cores (1-4 ou $5-8)$, conforme ilustrado na Tabela 3.

Tabela 3 - critérios para cálculo do tempo padrão

\begin{tabular}{|c|c|c|c|c|c|}
\hline Critério & Chapa & Espessura & Marca & Gravadora & Cores \\
\hline 1 & Jumbo & 0,76 & MacDermid & CDI Wide TermoFlex & $1-4$ \\
\hline 2 & Jumbo & 0,76 & MacDermid & CDI Wide TermoFlex & $5-8$ \\
\hline 3 & Jumbo & 0,76 & MacDermid & CDI Spark 5080 & $1-4$ \\
\hline 4 & Jumbo & 0,76 & MacDermid & CDI Spark 5080 & $5-8$ \\
\hline 5 & Retalho & 0,76 & MacDermid & CDI Wide TermoFlex & $1-4$ \\
\hline 6 & Retalho & 0,76 & MacDermid & CDI Wide TermoFlex & $5-8$ \\
\hline 7 & Jumbo & 1,14 & Dupont e MacDermid & CDI Wide TermoFlex & $1-4$ \\
\hline 8 & Média & 1,14 & Dupont e MacDermid & CDI Wide TermoFlex & $1-4$ \\
\hline 9 & Retalho & 1,14 & Dupont e MacDermid & CDI Wide TermoFlex & $1-4$ \\
\hline 10 & Jumbo & 1,14 & Dupont e MacDermid & CDI Wide TermoFlex & $5-8$ \\
\hline 11 & Média & 1,14 & Dupont e MacDermid & CDI Wide TermoFlex & $5-8$ \\
\hline 12 & Retalho & 1,14 & Dupont e MacDermid & CDI Wide TermoFlex & $5-8$ \\
\hline 13 & Jumbo & 1,14 & Dupont e MacDermid & CDI Spark 5080 & $1-4$ \\
\hline 14 & Jumbo & 1,14 & Dupont e MacDermid & CDI Spark 5080 & $5-8$ \\
\hline 15 & Média & 1,70 & Dupont e MacDermid & CDI Wide TermoFlex & $1-4$ \\
\hline 16 & Média & 1,70 & Dupont e MacDermid & CDI Wide TermoFlex & $5-8$ \\
\hline 17 & Retalho & 1,70 & Dupont e MacDermid & CDI Wide TermoFlex & $1-4$ \\
\hline 18 & Retalho & 1,70 & Dupont e MacDermid & CDI Wide TermoFlex & $5-8$ \\
\hline 19 & Média & 2,84 & Dupont & CDI Wide TermoFlex & $1-4$ \\
\hline 20 & Média & 2,84 & Dupont & CDI Wide TermoFlex & $5-8$ \\
\hline 21 & Retalho & 2,84 & Dupont & CDI Wide TermoFlex & $1-4$ \\
\hline 22 & Retalho & 2,84 & Dupont & CDI Wide TermoFlex & $5-8$ \\
\hline
\end{tabular}

Fonte: Elaborado pelos autores, 2012. 
A organização trabalha 17 horas diárias, assim, estima-se que sejam concedidos $15 \%$ do tempo total para fadiga (17 horas $\times 0,15=2,55$ horas), ou seja, o valor do fator de tolerância fica $2,55 / 17$ horas $=0,15$ para os tempos de processo variável. No caso dos tempos padrão de máquina foi considerado como fator de tolerância $10 \%$ do tempo total, assim, 17 horas $\mathrm{x}$ $0,10=1,70$, ou seja, o valor do fator de tolerância fica $170 / 17$ horas $=0,10$. Apesar da dependência direta da mão de obra, os tempos de máquinas somam mais de $50 \%$ do total do tempo médio de processo de gravação. Desse modo, velocidade do operador foi avaliada em $100 \%$.

A partir dos tempos médios de cronometragem e a velocidade do operador, foi possível calcular o tempo normal por meio da fórmula TN $=$ TCR $\times$ V. Na Tabela 4, por meio da fórmula TP $=\mathrm{TN} \times(1+\mathrm{FT})$, foi possível calcular o tempo padrão para cada critério estabelecido.

Tabela 4 - Tempo padrão por critério

\begin{tabular}{|c|c|c|c|}
\hline Critério & Tempo & Critério & Tempo \\
\hline Critério 1 & 3,5 & Critério 12 & 3,05 \\
\hline Critério 2 & 3,58 & Critério 13 & 3,68 \\
\hline Critério 3 & 3,61 & Critério 14 & 3,76 \\
\hline Critério 4 & 3,69 & Critério 15 & 3,35 \\
\hline Critério 5 & 2,89 & Critério 16 & 3,43 \\
\hline Critério 6 & 2,97 & Critério 17 & 3,08 \\
\hline Critério 7 & 3,58 & Critério 18 & 3,16 \\
\hline Critério 8 & 3,24 & Critério 19 & 3,66 \\
\hline Critério 9 & 2,97 & Critério 20 & 3,74 \\
\hline Critério 10 & 3,66 & Critério 21 & 3,39 \\
\hline Critério 11 & 3,32 & Critério 22 & 3,47 \\
\hline
\end{tabular}

Fonte: Elaborado pelos autores, 2012.

Ainda, no cálculo do tempo padrão, foi calculado o tempo padrão por etapa conforme disposto na Tabela 5. Cabe informar que foram anotadas pelos operadores de gravação do turno diurno e noturno, entre os dias doze de setembro e doze de outubro, as horas paradas programadas e não programadas e que o período citado acima corresponde à vinte e dois dias de trabalho. 
Ainda cabe informar que foi elaborada uma planilha para a anotação das horas paradas com os seguintes critérios: treinamento, reunião, manutenção corretiva e preventiva, falta de serviço, falta de matéria-prima etc., separadas por turno e por gravador.

\subsection{HORAS PARADAS PROGRAMADAS}

Neste capítulo serão abordadas as horas paradas programadas, cujo intuito é fornecer dados acerca da capacidade efetiva da organização. Nesse sentido, cabe colocar que Martins e Laugeni (2006) afirmam que a capacidade efetiva é o que o equipamento apresenta depois de descontar todas as horas técnicas paradas, que envolvem o tempo necessário para manutenção, preparação, processo, limpeza, entre outros.

Cabe também fundamentar o processo nas ideias de Slack et al., (2008) que definem que as perdas se dão em casos de mudanças de produtos, manutenção dos equipamentos e entre outros. Após deduzir essas perdas resta a capacidade efetiva da operação.

Em uma conversa informal com os funcionários do setor de gravação, quando questionados sobre a utilização das gravadoras, responderam que a gravadora CDI Wide TermoFlex é a gravadora mais utilizada e que a CDI Spark 5080 só é utilizada quando a ordem de serviço informar que o processo de gravação do arquivo deve ser em uma resolução maior do que a suportada na CDI Wide TermoFlex, ou também na situação de entrada de muitos serviços e a CDI Wide TermoFlex não conseguir suprir essa demanda. Assim, constatou-se que a CDI Wide TermoFlex atua em média quinze horas por dia e que a CDI Spark 5080 cerca de nove horas. 
A CAPACIDADE DE PRODUÇÃO COMO RECURSO GERADOR DE GANHOS

Tabela 5 - Tempo padrão por etapa

\begin{tabular}{|c|c|c|c|c|c|c|c|c|c|c|c|c|c|c|c|c|c|c|c|}
\hline \multicolumn{2}{|c|}{ ETAPA 1} & \multicolumn{2}{|c|}{ ETAPA 2} & \multicolumn{2}{|c|}{ ETAPA 3} & \multicolumn{2}{|c|}{ ETAPA 4} & \multicolumn{2}{|c|}{ ETAPA 5} & \multicolumn{2}{|c|}{ ETAPA 6} & \multicolumn{2}{|c|}{ ETAPA 7} & \multicolumn{2}{|c|}{ ETAPA 8} & \multicolumn{2}{|c|}{ ETAPA 9} & \multicolumn{2}{|c|}{ ETAPA 10} \\
\hline JUMBO & 0,26 & JUMBO 1 & 0,77 & 0,76 & 0,18 & 0,76 & 0,28 & 0,76 & 1,38 & 0,76 & 0,09 & 0,76 & 0,04 & JUMBO & 0,23 & $1-4$ & 0,06 & PACOTE & 0,14 \\
\hline MÉDIA & 0,18 & MÉDIA & 0,59 & 1,14 & 0,18 & 1,14 & 0,28 & 1,14 & 1,38 & 1,14 & 0,09 & 1,14 & 0,11 & MÉDIA & 0,14 & $5-8$ & 0,14 & & \\
\hline \multirow[t]{2}{*}{ RETALHO } & 0,20 & RETALHO & 0,37 & 1,7 & 0,18 & 1,7 & 0,37 & 1,7 & 1,38 & 1,7 & 0,09 & 1,7 & 0,13 & RETALHO & 0,07 & & & & \\
\hline & & JUMBO 2 & 0,87 & 2,84 & 0,24 & 2,84 & 0,46 & 2,84 & 1,38 & 2,84 & 0,09 & 2,84 & 0,28 & & & & & & \\
\hline
\end{tabular}

Fonte: Elaborado pelos autores, 2012. 
Na Tabela 6 estão apresentadas as horas paradas programadas que consistiram em treinamento realizado no horário da troca de turnos, sobre o uso dos equipamentos de proteção individual, cujo tempo foi de 01:30:00 minutos. As gravadoras necessitam de dez minutos diários para o aquecimento; a lavadora exige cinco minutos diários para limpeza do seu interior; e aquecimento do forno demora trinta minutos diários. Nesse período de marcações de horas paradas ocorreram cerca de seis horas diárias por falta de serviço, sendo o total de quatro horas durante o turno diurno e duas horas no turno noturno. Cabe ressaltar que a gravação depende da entrada de serviços nas fases anteriores à gravação. Assim, o pico de produção começa depois do meio-dia e aumenta até o final do dia.

Tabela 6 - Horas paradas programadas

\begin{tabular}{llll}
\hline Processos & Tempo Diário & Em Horas & Horas/Mês \\
\hline Aquecimento das gravadoras & $00: 10: 00$ & 0,1666 & 3,6667 \\
Limpeza da lavadora & $00: 05: 00$ & 0,0833 & 1,8333 \\
Aquecimento do forno & $00: 30: 00$ & 0,5000 & 11,000 \\
Treinamento & $00: 04: 09$ & 0,0682 & 1,5000 \\
Falta de Serviço & $06: 00: 00$ & 6,0000 & 132,00 \\
TOTAL & $\mathbf{0 6 : 4 9 : 0 9}$ & $\mathbf{6 , 8 1 8 2}$ & $\mathbf{1 5 0 , 0 0}$ \\
\hline
\end{tabular}

Fonte: Elaborado pelos autores, 2012.

Desse modo, para calcular a capacidade efetiva da organização é necessário tomar o total de horas trabalhadas diariamente em condições normais e deduzir as horas paradas programadas. Ou seja, 17 horas trabalhadas menos 6,82 de hora parada programada, que é igual à 10,18 horas.

\subsection{HORAS PARADAS NÃO PROGRAMADAS}

Neste capítulo, serão abordadas as horas paradas não programadas, que conforme Slack et al. (2008) consiste em deduzir também as perdas que não fazer partem do processo produtivo, a exemplo de problemas com a qualidade.

As horas paradas não programadas consistiram em duas quedas de energia (ocasiona a perda da chapa em gravação; é necessário iniciar uma nova gravação, tempo total de retrabalho 01:10:00, assim, dividido por vinte e dois dias médios de trabalho mensal, resulta 
em 00:03:18 minutos diários). Foi realizada uma reunião entre gerência e gravação no turno diurno, com duração de 01:30:00, assim, dividido por vinte e dois dias médios de trabalho mensal, resulta em 00:04:09 minutos diários. Também foram realizados três testes do fornecedor (que resultaram em 03:45:00, que dividido por vinte e dois dias médios de trabalho mensal, resulta em 00:10:23 minutos diários), e devido a um problema ainda não identificado ocorreu uma interrupção na gravadora CDI Spark 5080 (que resultou em 00:25:00 minutos de hora parada, que dividido por vinte e dois dias médios de trabalho mensal, resulta em 00:01:14 minutos diários). O tempo em horas corresponde ao total de horas diárias, e o tempo mensal corresponde ao tempo diário em horas multiplicado por vinte e dois dias. Conforme representado na Tabela 7:

Tabela 7 - horas paradas não programadas

\begin{tabular}{llll}
\hline Processos & Tempo Diário & Em Horas & Horas/Mês \\
\hline Queda de energia & $00: 03: 18$ & 0,0550 & 1,2100 \\
Reunião & $00: 04: 09$ & 0,0667 & 1,4674 \\
Teste Fornecedor & $00: 10: 23$ & 0,1731 & 3,8082 \\
Abortagem da gravadora & $00: 01: 14$ & 0,0206 & 0,4532 \\
TOTAL & $\mathbf{0 0 : 1 9 : 0 4}$ & $\mathbf{0 , 3 1 5 4}$ & $\mathbf{6 , 9 3 8 8}$ \\
\hline
\end{tabular}

Fonte: Elaborado pelos autores, 2012.

Desse modo, para calcular a capacidade real da organização é necessário tomar o total de horas trabalhadas diariamente em condições normais e deduzir as horas paradas não programadas. Ou seja, 10,18 horas trabalhadas menos 0,3154 de hora parada não programada, que é igual à 9,86 horas.

No próximo item será calculada a capacidade do projeto (consiste em tomar o total de horas disponíveis e dividir pelo tempo padrão de cada etapa), a capacidade efetiva (consiste em deduzir as horas paradas programadas do total de horas disponíveis e dividir pelo tempo padrão) e por fim a capacidade real (consiste em deduzir as horas paradas programadas e não programadas do total de horas disponíveis e dividir pelo tempo padrão), que vinculado às ideias de Moreira (2004) capacidade é a quantidade máxima de produtos e/ou serviços que podem ser produzidos numa unidade produtiva, em dado intervalo de tempo.

\subsection{CAPACIDADE DE PRODUÇÃO}


Neste item será apresentado o cálculo da capacidade de produção da organização, informação esta que propiciará o atendimento do objetivo geral deste trabalho. O cálculo da capacidade de produção do setor de gravação envolve conhecer a capacidade do projeto, capacidade efetiva e capacidade real. No cálculo da capacidade do projeto, verificou-se que a organização dispõe de dezessete horas diárias de trabalho, e baseado nisso calculou-se a capacidade de produção diária por etapa, tomou-se o tempo total de horas disponíveis e dividiu-se pelo tempo padrão da etapa. Assim, obteve-se a capacidade total em chapas.

Para o cálculo da capacidade efetiva, foi retirado do total de horas disponíveis o total de horas paradas programadas. Também calculada por etapa, verificou-se que a organização dispõe de 10,18 horas de trabalho diário, e baseado nisso calculou-se a capacidade de produção diária por etapa; tomou-se o tempo total de horas disponíveis e dividiu-se pelo tempo padrão da etapa.

Para calcular a capacidade real, que conforme Corrêa e Corrêa (2008) é a capacidade identificada após deduzir as perdas evitáveis (exemplo, quebra por falta de manutenção) e perdas inevitáveis (exemplo, troca de turno, mudança de produto), também calculada por etapa, verificou-se que a organização dispõe de 9,86 horas de trabalho diário, e baseado nisso calculou-se a capacidade de produção diária por etapa; tomou-se o tempo total de horas disponíveis e dividiu-se pelo tempo padrão de cada etapa.

O cálculo da capacidade de produção permitiu concluir que as gravadoras representam o gargalo de produção, que na visão de Ritzmann e Krajewski (2004), gargalo é uma operação que possui a menor capacidade efetiva de qualquer operação no processo, limitando a produção do sistema. Permite-se notar que a operação mais lenta determina a produção do processo, e nesse sentido, expandir a capacidade de produção está diretamente vinculada ao aumento da capacidade do gargalo.

Assim, na Figura 3 elaborou-se um demonstrativo do fluxo dos processos para a gravação das chapas até a sua finalização e sinalizou-se a restrição do sistema. Para obtenção da capacidade real, calculou-se a média das capacidades por etapa em que a divisão é por espessura da chapa e quantidade de cores; e nas etapas cujo critério é o tamanho da chapa, utilizou-se o valor da chapa jumbo. 
Figura 3 - Fluxo dos processos de gravação

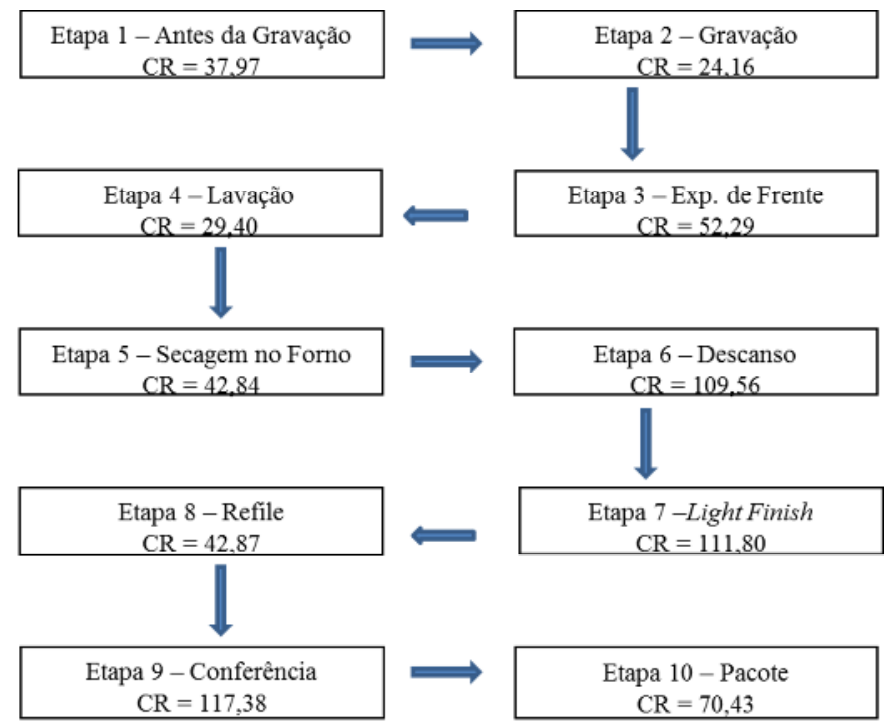

Fonte: Elaborado pelos autores, 2012.

Conforme Corrêa e Corrêa (2008), com o cálculo da capacidade de produção real e a capacidade do projeto é possível mensurar a porcentagem de utilização da unidade de produção e ainda infere que o resultado desse cálculo dá a ideia de quanto da capacidade do projeto está sendo utilizada baseada na capacidade real. Para a exposição dos cálculos foi utilizada a fórmula apresentada por Slack et al. (2008):

Figura 4 - Cálculo de eficiência

Utilização: Volume de produção real x 100

Capacidade do projeto

Fonte: Slack et al., (2008).

Ainda segundo Corrêa e Corrêa (2008), com os dados da capacidade real e a capacidade efetiva é possível mensurar a eficiência da unidade de produção, pois esse cálculo propicia identificar qual a real eficiência de projeto, afinal, ele deduz todas as perdas evitáveis e inevitáveis em relação à capacidade efetiva. Para a exposição dos cálculos foi utilizada a fórmula apresentada por Slack et al. (2008): 
Figura 5-Cálculo de eficiência

Eficiência: Volume de produção real x 100

Capacidade efetiva

Fonte: Slack et al., (2008).

Os valores informados na capacidade do projeto, efetiva e real, são referentes à quantidade de chapas jumbo, médias e retalhos que podem ser produzidas por dia levando em consideração a capacidade da restrição do sistema, sendo $58 \%$ de utilização e $97 \%$ de eficiência.

Os cálculos da porcentagem de utilização e eficiência demonstraram que com a comparação da capacidade de produção do projeto e a capacidade de produção real, há um grande número de horas paradas programadas por falta de serviço na organização. Fica evidente que a capacidade de produção poderá ser maior se essas horas paradas programadas por falta de serviço forem utilizadas para a produção. Assim existe a possibilidade de produzir mais com a mesma unidade produtiva existente não sendo necessário aumentar a estrutura atual para suprir um aumento na demanda.

No próximo item será feita uma comparação do desempenho padrão calculada com o desempenho atingido pela organização. Cabe ressaltar que para a capacidade real será considerada a capacidade total de gravação de chapas jumbo, devido ao fato desta capacidade apresentar a maior área de aproveitamento e no caso do desempenho atingido pela organização será considerada a área total gravada por dia do mês de setembro do ano de dois mil e onze, independente de ser chapa jumbo, média ou retalho.

\subsection{COMPARAÇÃO DO DESEMPENHO PADRÃO COM DESEMPENHO ATINGIDO}

O cálculo da capacidade de produção fornece informações acerca do desempenho padrão da organização, o que torna possível comparar com o desempenho atingido. Assim, desempenho é o grau no qual um sistema, físico ou econômico, atinge seus objetivos. Está associado à eficiência do sistema físico e à eficácia do sistema econômico (MARTINS; LAUGENI, 2006). 
A falta de demanda no período matutino gera períodos de ociosidade. Para diminuir o custo à organização, mantém apenas dois funcionários no primeiro turno. A maioria dos serviços é despachado após a meia-noite, assim, o pico de produção acontece no segundo turno, e para atender a demanda a organização dispõe do dobro de funcionários.

Agora é possível comparar o desempenho padrão com o desempenho diário atingido, conforme apresentado na Tabela 8. Para o cálculo foram somadas as capacidades da gravadora CDI Wide TermoFlex e a da CDI Spark 5080 à capacidade total em chapas jumbo, assim 12,81 +11,35 = 24,16 cuja área total de produção é de $623.482,62 \mathrm{~cm}^{2}$ por dia.

Tabela 8 - Comparação desempenho padrão com desempenho atingido

\begin{tabular}{lllll}
\hline \multicolumn{1}{c}{ Dia } & \multicolumn{1}{c}{ Desempenho Padrão } & \multicolumn{1}{c}{ Desempenho Atingido } & Diferença & $\%$ \\
\hline 1 & $623.482,62 \mathrm{~cm}^{2}$ & $333.432 \mathrm{~cm}^{2}$ & $290.050,62 \mathrm{~cm}^{2}$ & $-46,52 \%$ \\
2 & $623.482,62 \mathrm{~cm}^{2}$ & $335.207 \mathrm{~cm}^{2}$ & $288.275,62 \mathrm{~cm}^{2}$ & $-46,24 \%$ \\
5 & $623.482,62 \mathrm{~cm}^{2}$ & $265.455 \mathrm{~cm}^{2}$ & $358.027,62 \mathrm{~cm}^{2}$ & $-57,42 \%$ \\
6 & $623.482,62 \mathrm{~cm}^{2}$ & $553.775 \mathrm{~cm}^{2}$ & $69.707,62 \mathrm{~cm}^{2}$ & $-11,18 \%$ \\
8 & $623.482,62 \mathrm{~cm}^{2}$ & $301.315 \mathrm{~cm}^{2}$ & $322.167,62 \mathrm{~cm}^{2}$ & $-51,67 \%$ \\
9 & $623.482,62 \mathrm{~cm}^{2}$ & $561.333 \mathrm{~cm}^{2}$ & $62.149,62 \mathrm{~cm}^{2}$ & $-9,97 \%$ \\
12 & $623.482,62 \mathrm{~cm}^{2}$ & $258.612 \mathrm{~cm}^{2}$ & $364.870,62 \mathrm{~cm}^{2}$ & $-58,52 \%$ \\
13 & $623.482,62 \mathrm{~cm}^{2}$ & $392.066 \mathrm{~cm}^{2}$ & $231.416,62 \mathrm{~cm}^{2}$ & $-37,12 \%$ \\
14 & $623.482,62 \mathrm{~cm}^{2}$ & $387.213 \mathrm{~cm}^{2}$ & $236.269,62 \mathrm{~cm}^{2}$ & $-37,90 \%$ \\
15 & $623.482,62 \mathrm{~cm}^{2}$ & $372.787 \mathrm{~cm}^{2}$ & $250.695,62 \mathrm{~cm}^{2}$ & $-40,21 \%$ \\
16 & $623.482,62 \mathrm{~cm}^{2}$ & $301.965 \mathrm{~cm}^{2}$ & $321.517,62 \mathrm{~cm}^{2}$ & $-51,57 \%$ \\
19 & $623.482,62 \mathrm{~cm}^{2}$ & $308.400 \mathrm{~cm}^{2}$ & $315.082,62 \mathrm{~cm}^{2}$ & $-50,54 \%$ \\
20 & $623.482,62 \mathrm{~cm}^{2}$ & $607.763 \mathrm{~cm}^{2}$ & $15.719,62 \mathrm{~cm}^{2}$ & $-2,52 \%$ \\
21 & $623.482,62 \mathrm{~cm}^{2}$ & $342.132 \mathrm{~cm}^{2}$ & $281.350,62 \mathrm{~cm}^{2}$ & $-45,13 \%$ \\
22 & $623.482,62 \mathrm{~cm}^{2}$ & $23.315,62 \mathrm{~cm}^{2}$ & $-3,74 \%$ \\
23 & $623.482,62 \mathrm{~cm}^{2}$ & $600.167 \mathrm{~cm}^{2}$ & $178.389,62 \mathrm{~cm}^{2}$ & $-28,61 \%$ \\
26 & $623.482,62 \mathrm{~cm}^{2}$ & $445.093 \mathrm{~cm}^{2}$ & $52.262,62 \mathrm{~cm}^{2}$ & $-8,38 \%$ \\
27 & $623.482,62 \mathrm{~cm}^{2}$ & $571.220 \mathrm{~cm}^{2}$ & $134.449,62 \mathrm{~cm}^{2}$ & $-21,56 \%$ \\
28 & $623.482,62 \mathrm{~cm}^{2}$ & $489.033 \mathrm{~cm}^{2}$ & $344.267,62 \mathrm{~cm}^{2}$ & $-55,22 \%$ \\
29 & $623.482,62 \mathrm{~cm}^{2}$ & $279.215 \mathrm{~cm}^{2}$ & $232.781,62 \mathrm{~cm}^{2}$ & $-37,34 \%$ \\
30 & $623.482,62 \mathrm{~cm}^{2}$ & $390.701 \mathrm{~cm}^{2}$ & $90.023,62 \mathrm{~cm}^{2}$ & $-14,44 \%$ \\
Total & $13.716 .617,73 \mathrm{~cm}^{2}$ & $533.459 \mathrm{~cm}^{2}$ & $5.086 .274,73 \mathrm{~cm}^{2}$ & $-37,08 \%$ \\
\hline Fon & $8.630 .343 \mathrm{~cm}^{2}$ & & \\
\hline
\end{tabular}

Fonte: Elaborado pelos autores, 2012.

Assim, nota-se que o pior dia de aproveitamento da capacidade de produção foi no dia doze de setembro, cujo aproveitamento foi de $-58,52 \%$, e o melhor dia foi dia vinte de setembro, cujo aproveitamento foi de $-2,52 \%$, o que permite confirmar que a produção atual está aquém do que se pode realmente produzir. 


\section{CONSIDERAÇÕES FINAIS}

Primeiramente cabe ressaltar que este estudo não deve terminar aqui. Sugere-se que desenvolva-se uma continuação dessa pesquisa, explorando com mais profundamente temas como: otimizar a capacidade produtiva da restrição do sistema, bem como, propor estratégias para diminuir as horas paradas programadas por falta de serviço.

A organização que conhece a sua capacidade produtiva tem os dados necessários para identificar se possui capacidade produtiva suficiente para o atendimento da demanda existente, assim como, o conhecimento necessário de quanto deve ser investido para suprir um possível aumento e ainda determinar a capacidade ociosa que está deixando de ser utilizada.

Num primeiro momento foi desenvolvido o referencial teórico cujo objetivo é fundamentar a teoria com a prática da organização. No segundo momento foram descritas as atividades que compreendem o processo de gravação das chapas de fotopolimero. Para cada etapa foram tomadas cem medições dos tempos de execução a fim de propiciar informações para o cálculo do tempo padrão. Em seguida foram identificadas as horas paradas programadas e não programadas, e na sequência foi calculada a capacidade do projeto, efetiva e real, bem como as porcentagens de eficiência e utilização. Ainda foi feito um quadro de comparação do desempenho atingido com o desempenho padrão.

Dessa forma, o desenvolvimento deste estudo atingiu o seu objetivo geral ao identificar a capacidade de produção do setor de gravação da matriz, assim como, os objetivos específicos que propiciaram a sustentação para a solução da questão problema, cujo intuito era vincular a capacidade de produção do setor de gravação à geração de ganhos. Ficou evidente que as horas paradas programadas e não programadas anotadas pelos operadores de gravação podem ser melhoradas, e que a presença de um profissional para a área de planejamento e controle de produção é imprescindível para otimizar as horas ociosas. A partir disso, sugere-se à organização que nomeie um profissional como coordenador desse setor, tendo em vista que os responsáveis pelo atendimento a clientes 
também fazem as programações de entradas dos serviços na gravação, o que muitas vezes compromete a própria produção, pois os operadores não têm o conhecimento da urgência de determinados serviços e acabam produzindo à medida que o mesmo é liberado para a gravação. Assim, um profissional experiente e com conhecimento técnico terá subsídios para otimizar a capacidade ociosa do primeiro turno e melhor programar a demanda do turno noturno.

Os pressupostos que deram início a esta pesquisa foram confirmados, pois ficou evidente que a organização não tinha conhecimento da sua real capacidade de produção e que a partir dos resultados desta pesquisa, torna-se necessário a implantação de um setor para planejamento e controle da produção, pois agora se tem informações que permitem otimizar a produção e atender com mais embasamento a demanda existente, podendo então melhorar os custos de fabricação, contribuindo para a geração de ganhos.

A partir disso, a investigação teve como resultado a identificação da restrição do sistema de produção, que está relacionada à capacidade de produção das gravadoras. Também foi identificado que as horas programadas por falta de serviço acarretam o comprometimento da capacidade de produção e que a organização pode encontrar meios para utilizar essa capacidade ociosa para otimizar os ganhos, pois aproveita melhor o tempo e assim pode melhorar o preço. Hora ociosa gera custo para a empresa. Então, diminuir os custos está inter-relacionado com eliminar as horas ociosas, assim é possível melhorar o preço, aumentar as vendas e manter o mesmo custo fixo.

Ainda, em caso de aumento da demanda, a organização pode optar por um terceiro turno, sem a necessidade de comprar mais equipamentos, apenas contratar mais mão de obra.

Assim, o conhecimento adquirido por meio da pesquisa é relevante para a empresa estudada, pois possibilitou a identificação da capacidade produtiva real, bem como identificação dos gargalos de produção. Outro ponto de destaque do estudo é a demonstração da aplicabilidade de ferramentas administrativas bastante difundidas, cuja utilização é adotada por muitas organizações que são referências em seus ramos de atuação. 
Para a área de gestão de operações, o estudo tem sua relevância a partir do momento que mostra para organização a importância de se conhecer a sua real capacidade de produção, para que esta possa se posicionar de maneira mais prática diante de seus clientes, disponibilizando informações de prazo de entrega, bem como, capacidade de atendimento ou não de um determinado volume no prazo desejado por esse cliente. Essas informações devem ser as mais confiáveis possíveis, uma vez que poderão se transformar em uma vantagem competitiva para a organização à medida que mantém a confiança do cliente em relação a sua marca e seu produto.

\section{PRODUCTION CAPACITY AS A REMEDY FOR PROFIT GENERATOR}

\section{ABSTRACT}

The gradual growth of the flexography market generates different expectations in organizations of this segment. To ensure the fulfillment of the demand and ensure its market share, it is important to know the real productive capacity of its plant. The research aimed to identify the capacity of the sector recording a stereotype workshop the city of Blumenau/SC. Was defined as qualitative research, objective descriptive and as in the investigation, the research is characterized as action research. Data were collected from the daily monitoring of the processes performed in the recording industry, observing the development of activities, recording information in reports and informal interviews with employees. Thus, the data were triangulated and presented in tables and figures. The results of this investigation was identified the bottleneck of production as well as the existence of hours shutdowns for lack of service, and made suggestions to optimize production capacity, as well as strategies to increase earnings.

Keywords: Flexography. Standard Time. Productive Capacity. System Restriction. 


\section{REFERÊNCIAS}

ANDERSON, E.W.; FORNELL, C.; RUST, R.T. A Customer satisfaction, productivity, and profitability: differences between goods and services. Marketing Science, vol. 16, n. 2, p. 129-45, 1997.

BARNES, Louis B., et al. Logística: essencial para a estratégia. São Paulo: Nova Cultural, 1986.

BERGER, A. Continuous improvement and kaizen: standartization and organizational designs. Integrated Manufacturing Systems, v. 8, n. 2, p. 110-117, 1997.

BESSANT, J.; FRANCIS, D. Developing strategic continuous improvement capability. International Journal of Operations \& Production Management, v. 19, n. 11, p. 1106-1119, 1999.

BOWEN, D.; SIEHL, C.; SCHNEIDER, B. Developing service-oriented manufacturing. In: Kilmann, I. (org.). Making Organizations Competitive. Jossey-Bass: San Francisco. 1991, p. 397-418.

CORRÊA, H.; CORRÊA, C. Administração de produção e operações: manufaturas e serviços: uma abordagem estratégica. São Paulo: Atlas, 2008.

GADIESH, O.; GILBERT, J.L. A Profitt pools: a fresh look at strategy. Harvard Business Review, v. 76, n. 3, p. 139-47, 1998.

GIL, A. C. Métodos e técnicas de pesquisa social. São Paulo: Atlas, 2006.

HESKETT, J.L.; SASSER, W.E.; SCHLESINGER, L.A. The Service Profit Chain. Free New York: Press, 1997.

IMAI, M. Kaizen: the key to japanâs competitive success. New York: Random House, 1986.

JOHNSTON, Robert. Administração de operações de serviço. São Paulo: Atlas, 2002.

KNECHT, T.; LESZINSKI, R.; WEBER, F. A Memo to a CEO. The McKinsey Quarterly, v. 4, p. 7986, 1993.

LOJO, M. Contracting of high-technology industrial services. Tese - Doutorado (Sloan School of Management). Massachusetts Institute of Technology, Cambridge, MA, 1997.

LEEDE, J.; LOOISE, J. K. Continuous improvement and the mini-company concept. International Journal of Operations \& Production Management, v. 19, n. 11, p. 1188-1202, 1999. 
MARTINS, P. G.; LAUGENI, F. P. Administração da produção. São Paulo: Saraiva, 2006.

MOREIRA, D. A. Administração da produção e operações. São Paulo: Pioneira Thomson Learning, 2004.

POTTS, G.W. Exploiting your product's service life cycle. Harvard Business Review, v. 66, n. 5, p. 32-5, 1988.

QUINN, J.B. Intelligent Enterprise. New York: Free Press, 1992.

QUINN, J.B.; DOORLEY, T.L.; PAQUETTE, P.C. Beyond products: services-based strategy. Harvard Business Review, vol. 68, n. 2, p. 58-67, 1990.

RICHARDSON, Roberto Jarry. Pesquisa social: métodos e técnicas. São Paulo: Atlas, 1989.

RITZMAN, Larry P.; KRAJEWSKI, Lee J. Administração da Produção e Operações. 1. ed. São Paulo: Prentice Hall, 2004.

SAVOLAINEN, T. Cycles of continuous improvement: realizing competitive advantages through quality - a longitudinal case study. Enschede, Netherlands: Twente University Press, 1998.

SLACK, Nigel et al. Administração da Produção. 2. ed. São Paulo: Atlas, 2008.

THIOLLENT, Michel. Crítica metodológica, investigação social e enquete operaria. São Paulo: Polis, 1987.

WISE, R.; BAUMGARTNER, P. Go downstream: the new imperative in manufacturing. Harvard Business Review, v. 77, n. 5, p. 133-41, 1999. 